\title{
Aggregate Dynamics in an Evolutionary Network Model
}

\author{
Adrian M. Seufert ${ }^{1}$, Frank Schweitzer ${ }^{2}$ \\ 1 Institute for Theoretical Physics, Technical University of Berlin, Harden- \\ berg Str. 36, D-10623 Berlin, Germany \\ 2 Chair of Systems Design, ETH Zurich, Kreuzplatz 5, CH-8032 Zurich, \\ Switzerland, Corresponding author: fschweitzer@ethz.ch
}

\begin{abstract}
We analyze a model of interacting agents (e.g. prebiotic chemical species) which are represended by nodes of a network, whereas their interactions are mapped onto directed links between these nodes. On a fast time scale, each agent follows an eigendynamics based on catalytic support from other nodes, whereas on a much slower time scale the network evolves through selection and mutation of its nodesagent. In the first part of the paper, we explain the dynamics of the model by means of characteristic snapshots of the network evolution and confirm earlier findings on crashes an recoveries in the network structure. In the second part, we focus on the aggregate behavior of the network dynamics. We show that the disruptions in the network structure are smoothed out, so that the average evolution can be described by a growth regime followed by a saturation regime, without an initial random regime. For the saturation regime, we obtain a logarithmic scaling between the average connectivity per node $\langle l\rangle_{s}$ and a parameter $m$, describing the average incoming connectivity, which is independent of the system size $N$.
\end{abstract}

Keywords: directed network, evolution, scaling laws

PACS Nos.: 89.75.Fb, 89.75.Da, 87.23.Kg, 64.60.Cn, 05.65.+b

\section{Introduction}

Many evolutionary processes in physical, biological or economic systems involve elements of selfreproduction and catalytic interactions. In his pioneering work, Eigen [3] pointet out their relevance for the prebiotic evolution of macromolecules, which lead to the theory of the hypercycle (see also [4]). The hypercycle can be seen as a paragon of a network of cooperating agents [7] (e.g. chemical or biological species), which counterbalances the effect of aggressive self-replication. While the latter one just leads to the survival of only 
one species - "survival of the fittest" - the dependence on catalytic interaction with other species also ensures the survival of the others and, hence, a coexistence of agents with very different "fitness" levels.

Recently, the hypercycle concept has been investigated in a modified setting, which combines the original idea of catalytic interactions with an external dynamics of the network representing the interaction structure. Inspired by earlier work [5, 26] Jain and Krishna [9] have focussed on the emergence of so-called autocatalytic sets (ACS) among agents, which do not self-replicate individually, but only replicate by means of the help of others. An ACS is then a cooperative structure, where different agents interact in such a way that the links representing these interactions form a closed cycle in terms of the network structure. Once an ACS appears, it boosts the replication of the agents involved, which leads to a larger growth or "output" of those agents involved in the ACS. It further allows other agents not directly part of the ACS but only linked to it, to still benefit from it as freeloaders.

Because such a catalytic replication dynamics eventually leads to a stationary state, Jain and Krishna 9] have added a disturbance of the interaction network in terms of a so-called "extremal dynamics" [1]. There, the least performing agent, i.e. the one with the lowest output, is - together with its links to other agents - replaced by a new agent that is linked to the existing interaction network in a random way. This network dynamics occurs on a much slower time scale compared to the agent dynamics itself. It ensures (i) that the dynamics of the system of agents does not get stuck in an equilibrium state, and (ii) may allow for "evolutionary" scenarios towards a better performance of the whole system.

Our work, discussed in the following, is based on the model of Jain and Krishna (JK) described above (see also [10]). In Chapter 2 we explain the dynamics and our numerical implementation of the JK model in more detail. In Chapter 3 we reproduce some important features of the model behavior, such as the emergence of the ACS and the crashes and recoveries in the network structure, by means of computer simulations that elucidate the network evolution. In Chapter 4 we extend our investigations to the aggregate behavior of the system, which to our knowledge was not investigated before. In particular, we show that the crashes and recoveries in single network realizations are smoothed out, so that the average evolution can be described by a saturation dynamics. We further obtain, by means of computer simulations, a logarithmic scaling function for the average connectivity per node dependent on the average incoming connectivity (which is a measure for the catalytic interaction). In Chapter 5 we summarize these findings and point to further interesting extensions of the model. In particular, we already mention the relation to recent network models for social and economic applications. [22, 8, 14, 12, 18] 


\section{The Model}

\subsection{Population and Network Dynamics}

The model discussed in the following was originally developed in the context of the "origin of life problem": the observation that something as structurally complex as a living cell was able to form, parting from a random mix of chemical components in a prebiotic "broth" [3, 26] on earth four billion years ago.

For a modelling approach, we consider a set of $N$ prebiotic chemical species, each of them characterized by a population $y_{i} \geq 0(i=1, \ldots, N)$. The dynamics of the variables $y_{i}$ shall be governed by the following equation:

$$
\dot{y}_{i}=\sum_{j}^{N} c_{i j} y_{j}-\phi y_{i} .
$$

$\phi$ is assumed to be a constant dilution flux, resulting e.g. from a natural movement of raw material out of the system (say through flood or tides). The $c_{i j}$ are the kinetic coefficients that describe the replication of species $i$ resulting from binary interactions with other species $j$. For simplicity, only $c_{i j} \in\{0,1\}$ is assumed. $c_{i j}=1$ represents a growth process of species $i$ due to the presence of species $j$ that acts as a catalysor only. Negative values of $c_{i j}$ would indicate inhibitory processes that are neglected here. Further, self-replicating species are not allowed, which means $c_{i i}=0$ for all $i$.

In a first approximation $\phi$ can be set to zero. This results in a linear dynamical system of coupled first-order differential equations in the populations $y_{i}$. In vector notation this reads:

$$
\dot{\mathbf{y}}=C \cdot \mathbf{y}
$$

where $C$ is the matrix containing all kinetic coefficients $c_{i j}$. The solution of the set of equations (2) depends on the properties of the matrix $C$ and has the general form:

$$
\mathbf{y}(t)=e^{C t} \mathbf{y}_{0}
$$

representing an exponential increase in time of the population vector. To avoid the problem of exploding populations we consider the vector of relative populations

$$
x_{i}=\frac{y_{i}}{\sum_{j} y_{j}} ; \quad \sum_{j} x_{j}=1
$$

Rewriting Eq. (1) by means of Eq. (41) gives us the relative population dynamics:

$$
\dot{x}_{i}=\sum_{j}^{N} c_{i j} x_{j}-x_{i} \sum_{k, j}^{N} c_{k j} x_{j} .
$$


Equation (5) has the property of preserving the normalization of $\mathbf{x}$. Henceforth we will always refer to the population vector as $\mathbf{x}$ and refer to the corresponding population dynamics of Eq. (5). The dilution flux $\phi$ disappears in this transformation (as long as it is assumed to be equal for every species) which gives us another reason to set it arbitrarily to zero in Eq. (11).

So far, we have discussed the dynamics of interacting species, where the interaction is described by the matrix $C$ that contains the elements $c_{i j}$ in terms of 0 and 1 . This dynamics can be interpreted as a catalytic network [11, 13, 20, 21] where the different species $i$ are represented by nodes, and their interaction by links between these nodes, cf. Fig. (1). More precisely,

$$
\begin{gathered}
c_{i j}=\left\{\begin{array}{l}
1 \rightarrow \text { species } j \text { catalyzes species } i \\
0 \rightarrow \text { nothing happens }
\end{array}\right. \\
C=\left(\begin{array}{llll}
0 & 0 & 1 & 0 \\
1 & 0 & 0 & 0 \\
0 & 1 & 0 & 1 \\
0 & 1 & 0 & 0
\end{array}\right)
\end{gathered}
$$

Figure 1: A directed graph consisting of 4 nodes (species) and 5 links (interactions). Left: the corresponding interaction matrix

The matrix containing the catalytic interactions $c_{i j}$ is called the adjacency matrix. The network of interactions is modeled on a directed graph, which means that the adjacency matrix is not generally symmetric: $c_{i j} \neq c_{j i}$. It should be noted that the matrix $C$ represents a linear dynamical system in Eq. (1), and, simultaneously a directed graph of interactions. This means that $C$ acquires both structural and dynamical significance.

So far, the dynamics given by Eq. (5) are considered for a fixed configuration of the matrix $C$, which translates into a fixed network structure. In the following, we want to introduce a dynamics for the network itself, which means an additional element in our model, where the population dynamics is given by Eq. (5). In agreement with [9], our main assumption is here that the two different dynamics, the population dynamics and the network dynamics, occur on two different time scales. More precisely, it is assumed 
that the population dynamics is fast and relaxes into a quasi-stationary state (or attractor) soon, whereas the network dynamics occurs on a much slower time scale, and only happens after the population dynamics has reached its attractor. Hence, we are able to separate these two different time scales in our computer simulations, described below, and will measure time as "network time", i.e. in steps $n$ of the network modification.

For the network dynamics, we assume that the initial network of chemical reactions is a randomly generated graph: each $c_{i j}(i \neq j)$ equals 1 with probability $p$ and 0 with probability $(1-p)$. Each node contributes on average $m=p(N-1)$ links to the network, and average total connectivity is $N \cdot m$. The parameter $m$ is called average incoming connectivity.

The rules for the network evolution are the following:

- After a given time $T$ at which the population dynamics Eq. (5) is expected to have relaxed to its attractor configuration, the least fit species, i.e., the one with the smallest $x_{i}(T)$, is determined and removed from the network along with all its links. If there are different species with the same smallest values, then from these one species is chosen at random.

- A new species is added to the network with some small initial population $x_{0}$. The new species will take the place of the old one (it gets the same label), and is randomly rewired to the network with the same probability $p$ for establishing links that have been used in the initial network distribution. Incoming and outgoing nodes are statistically similar.

- Finally, the vector of relative populations $\mathbf{x}$ is re-normalized with the new node.

These rules for the network evolution are intended to capture two key features: natural selection, in this case, the extinction of the weakest; and the introduction of novelty. Both of these can be seen as lying at the heart of natural evolution. The particular form of selection used in this model has been inspired by the "extremal dynamics" of Bak and Sneppen [1] In the usual setting of a mutation/selection scheme, one has to realize that the two parts of the dynamics act on different levels: selection occurs on the level of the agents - i.e. removal of the least fittest prebiotic species, whereas mutation occurs on the level of the agent interaction, i.e. in terms of a random rewiring of a new node.

\subsection{Numerical Implementation}

For the numerical implementation we have to deal with the two different time scales of the model, introduced above. Here, we exploit the fact that the population dynamics occurs 
on a short time scale and relaxes fast into an attractor, whereas the network dynamics occurs on a much slower time scale and can thus be separated.

The key insight leading to the numerical implementation of the population dynamics is to see that the fixed points of the system described by Eq. (5) correspond to the eigenvectors of the adjacency matrix $C$. Since $C$ is a real non-negative matrix, the Perron-Frobenius Theorem tells us that the largest eigenvalue of $C$ is real and positive [2]. Furthermore, the corresponding Perron-Frobenius eigenvector is the only eigenvector with purely positive entries, and represents the unique asymptotically stable attractor of the population dynamics. One way to see this is to imagine our initial population vector $\mathbf{x}_{0}$ as a linear combination of all eigenvectors of $C$. Then Eq. (2) tells us that for large times $t$, the component of $\mathbf{x}_{0}$ corresponding to the largest eigenvalue will dominate all others as $\mathbf{x}(t)=e^{\lambda_{1} t} \mathbf{x}_{\lambda_{1}}$ where $\mathbf{x}_{\lambda_{1}}$ is the Perron-Frobenius eigenvector.

In order to find the attractor configuration of the population dynamics, we adopt the power method, meaning that the vector of initial populations $\mathbf{x}_{\mathbf{0}}$ can be expanded in terms of eigenvectors of $C: \mathbf{x}_{0}=a_{1} \mathbf{x}_{\left(\lambda_{1}\right)}+\cdots+a_{N} \mathbf{x}_{\left(\lambda_{N}\right)}$ with $\left|\lambda_{1}\right|>\left|\lambda_{2}\right| \cdots>\left|\lambda_{N}\right|$. Then

$$
C \mathbf{x}_{0}=a_{1} \lambda_{1} \mathbf{x}_{\lambda_{1}}+\cdots+a_{N} \lambda_{N} \mathbf{x}_{\lambda_{N}}
$$

and repeated iteration of this process yields

$$
C^{k} \mathbf{x}_{\mathbf{0}}=a_{1}^{k} \lambda_{1}^{k} \mathbf{x}_{\lambda_{1}}+\cdots+a_{N}^{k} \lambda_{N}^{k} \mathbf{x}_{\lambda_{N}}
$$

It is clear that for large $k$, the largest eigenvalue will dominate all others and thus $C^{k} \mathbf{x}_{\mathbf{0}}$ will approach $\mathbf{x}_{\lambda_{\mathbf{1}}}$. One obvious problem persists though: if $C$ has an eigenvalue that is equal in magnitude to $\lambda_{1}$ but with the opposite sign, then this method will give us incorrect results if the number of iterations is even. To be certain that we have reached the eigenvector corresponding to the positive eigenvalue, we add a $N \times N$ unit matrix to $C$. This increases all the eigenvalues of $C$ by one while leaving the corresponding eigenvectors unchanged. In this way we can be sure that the attractor we have reached is the correct one.

We point out that this method allows us to find the attractor configuration of Eq. (5) directly by exploiting the algebraic properties of the adjacency matrix $C$. This greatly reduces the costs in computation time and resources compared to a standard numerical method for solving systems of differential equations like the Runge-Kutta method. A number $\sim N$ of iterations usually suffices to get reasonably close to the attractor. The number of operations for reaching the attractor is thus of order $O\left(N^{2}\right)$.

The numerical implementation of the network dynamics is straightforward with respect to the rules given in Chapter (2.1). At each time step $n$ on the network time scale, we 
have to determine the smallest element, say $j$, from the population vector characterizing the attractor (using standard algorithms). The respective node is rewired at random with a linking probability $p$. This happens in two stages: first, node $j$ attempts to link itself to all other nodes $i \neq j$, which determines the outgoing links, secondly, the incoming links of node $j$ are set by each node $i \neq j$ attempting to link to $j$. The rewiring of node $j$, which involves $\sim 2 N$ steps, changes the adjacency matrix $C$ that will then feed back to the population dynamics. I.e. after the rewiring, we have to determine the new attractor of the population dynamics as explained above. Since we know that the population dynamics always converges to the unique attractor, the initial conditions for that process do not matter, so we always choose $\mathbf{x}_{0}$ as the starting point.

For the computer simulations discussed in the following, the time scale of the model is given by the time scale of the network dynamics only, i.e. abbreviated by $n$.

\section{Results of Computer Simulations}

\subsection{Evolution of Network Structure}

In Figs. (22)-(41) we show, by means of single snapshots, three phases of development of the network according to the dynamics described above. In the example given, the network consists of 100 nodes and was generated using an average random connectivity value $m=0.25$. The structural properties in each of these phases will be discussed in the following chapters.

The first of the graphs, Fig. 2, depicts a typical network in an early stage of evolution. The network is sparsely connected and contains many singletons. Typical structures are long chains and simple trees. The nodes located at the end of the longest chains will usually dominate all other nodes population-wise. In the second graph, Fig. 3, evolution created a new kind of structure: a cycle consisting of six nodes (dark nodes in the picture). Because they collectively catalyze each other, the members of the cycle will always have non-zero population in the attractor configuration. Thus, as long as there are singletons or separate chains and trees in the system, members of the cycle remain "immune" to selection. This immunity extends to all sets of nodes that have an incoming link coming from the cycle. These nodes form a parasitic periphery around the core formed by the cycle. Finally, the graph in 4 shows a fully connected network. The network is organized around a complex core consisting of several cycles, from which the periphery sprouts outwards. In this configuration, every node has at least one incoming link from some other node in the network. 


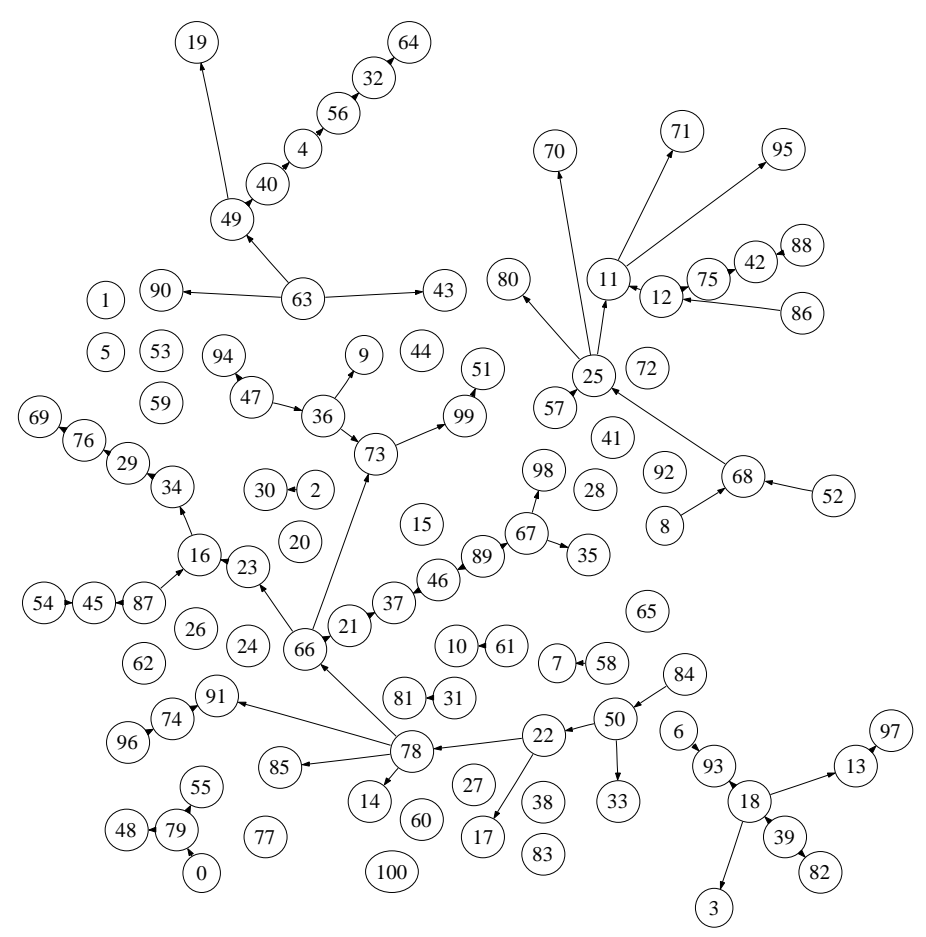

Figure 2: Network structure in the random phase $(n=800)$ : several chains and trees exist, but no supporting structure (i.e. autocatalytic set) has yet emerged. Parameters: $N=100, m=0.25$.

\subsection{The Autocatalytic Set}

The cycle of nodes present in Fig. (3) together with all the chains and trees parting at some member-node of the cycle is an example of an autocatalytic set or ACS. An ACS is defined as a subgraph whose every node has at least one incoming link from another node that belongs to the same subgraph. The simplest ACS is a two-cycle. The following correspondences between ACSs and $\lambda_{1}$ have been found [9]:

(i) An ACS always contains a cycle.

(ii) If a graph has no ACS then $\lambda_{1}=0$ for that graph.

(iii) If a graph has an ACS then $\lambda_{1} \geq 1$.

(iv) If $\lambda_{1} \geq 1$, then the subgraph spanned by nodes $i$ for which $\mathbf{x}_{\left(\lambda_{1}\right), i}>0$ is an ACS.

Cycles and structures of interlocking cycles represent irreducible subgraphs. The PerronFrobenius eigenvalue of a network is equal to the Perron-Frobenius eigenvalue of its dominant irreducible subgraph. The irreducible subgraph of a network that gives rise to the 


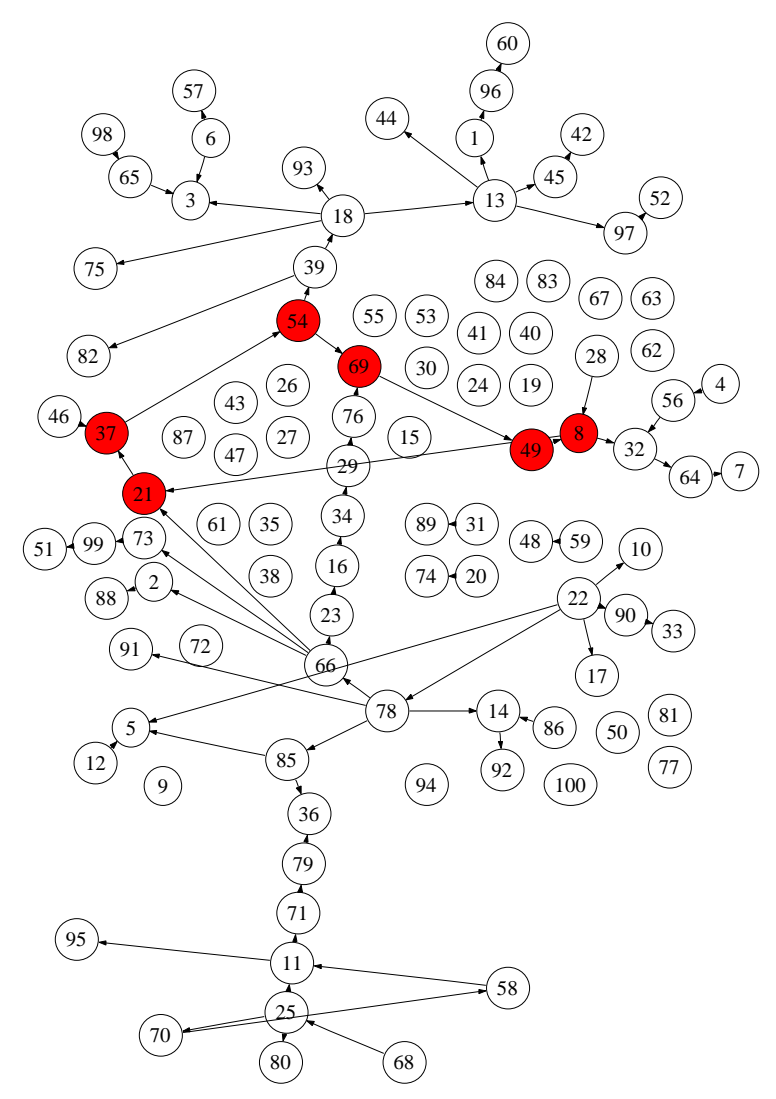

Figure 3: Network structure after a first autocatalytic set emerges $(n=973)$ : the core of the ACS (dark nodes) consists of a six-cycle, which has different "parasitic" chains (e.g. the chain of nodes 32, 64, and 7. Parameters see Fig. 2 .

largest $\lambda_{1}$ is called the core of the network (or alternatively the core of the dominant ACS). The importance of the ACS in this system is the following: once this structure appears, all the member nodes of the ACS have non-vanishing populations in the attractor. Because of that, they dominate all other nodes that are outside of the ACS. The ACS is thus robust against random mutations of its members. A small ACS appearing randomly in this system acts like a seed for the emerging network. It "attracts" the other nodes, since these can survive only by becoming members of the ACS. Finally, the fully developed network at the end of the evolution consists of a single, giant ACS.

\subsection{Network Connectivity}

In order to quantitatively characterize the network evolution, the average connectivity per node in the network is a useful observable. We denote this observable by $\langle l\rangle=l / N$, 


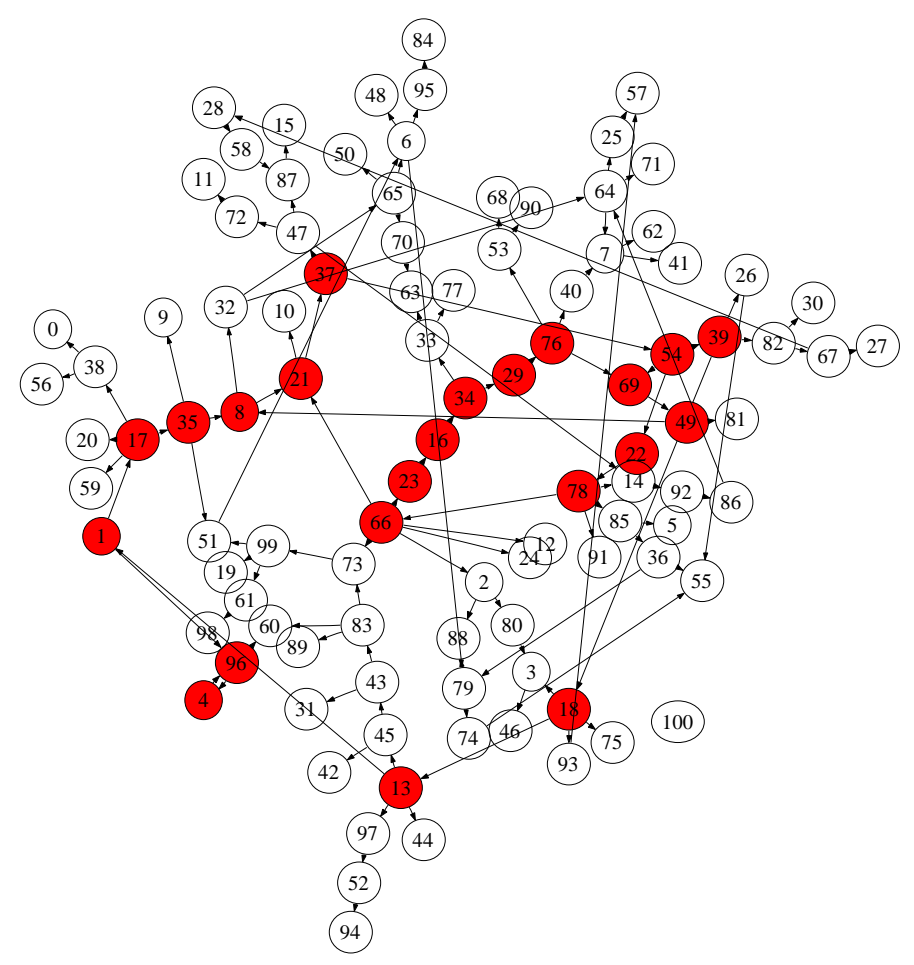

Figure 4: Network structure after the ACS spans the whole graph $(n=1290)$ : the core has been expanded as well, but the original six-cycle still exists. Parameters see Fig. 2.

where $l$ is the total number of links in the network. Typically we will choose $m$ to be in the range $(0,1)$, so in the early phase of network evolution the graph is very sparse. For example, a network consisting of 100 nodes and $m=0.25$ will have on average $2 \cdot m=50$ links.

Fig. 5 shows three typical runs for the evolution of $\langle l\rangle$ over network time $n$, which confirm the findings of [9] (Fig. 1). During each time-step, the network undergoes a transformation due to the selection and random re-wiring of a node. In effect, we have a different network at every step, and we count and plot the total number of links in each network divided by the number of nodes. The runs exhibits three distinct phases. The graphs in Figs. $2-4$ were chosen to exemplify each of these phases. In the first phase (random phase) the number of links hovers around the average expected number of $2 N \cdot m$. This phase is followed by one of exponential increase in the number of links (exponential phase). Finally, the number of links stabilizes at a much higher level (steady phase). A fundamental structural change in the network occurs between the random phase and the exponential phase: the emergence of an ACS. Once it appears in the network, it remains there until one-by-one all remaining nodes link themselves into the ACS and it eventually spans the whole graph. 


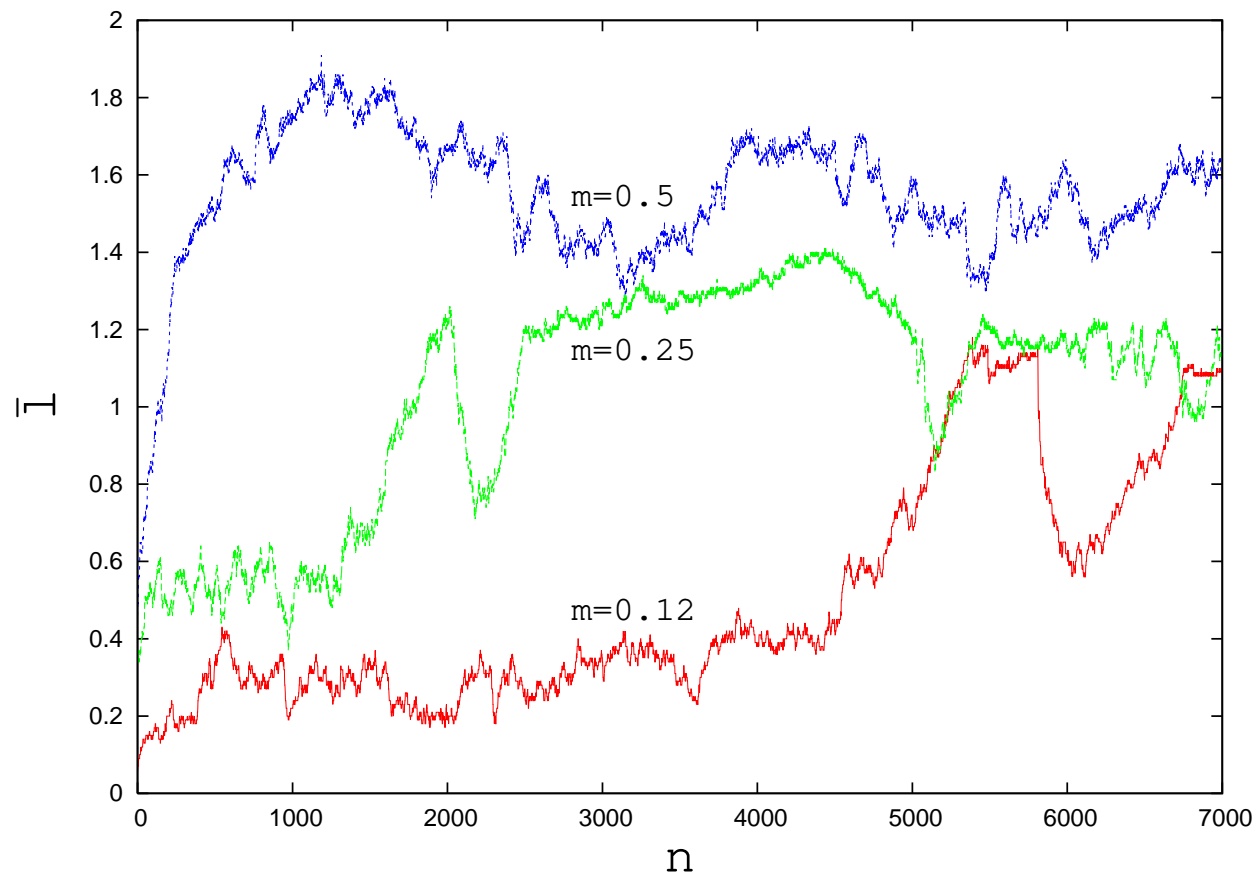

Figure 5: Average number of links, $\langle l\rangle$ evolving over network time, $n$, for three values of the parameter $m$. System size $N=100$

Only then will members of the ACS themselves be eligible for selection and mutation. The steady phase is reached when all the nodes in the network are members of the ACS. It is characterized by the fact that the mutating node has, on average, the same total number of links (namely $2 m$ ) as its replacement. Once the ACS engulfs the whole set of nodes, its members become eligible for selection. When a keystone species, i.e. a species critical to the support of the system, happens to be selected [9], the network loses its supporting structure and larger crashes occur. In Fig. 5 that is the case at $n \approx 5800$ for the $m=0.12$ run, and at $n \approx 2000$ and $n \approx 5000$ for the $m=0.25$ run. In longer runs, the number of crashes of this sort can be quite substantial, including crashes that completely destroy the ACS forcing the system to "start over". It is clear that for any $m>0$ an ACS will always emerge eventually. The interesting point is that, although we start from a random graph and introduce random mutations, the network resulting from the graph-spanning ACS is highly non-random. We repeat here that the probability of a graph with $N$ nodes and an average of $m$ links per node being an ACS is given by:[10]

$$
P=\left[1-\left(1-\left[\frac{m}{(N-1)}\right]\right)^{N-1}\right]^{N}
$$

which declines exponentially with $N$ when $m \sim O(1)$. 


\section{Network Structure at the Aggregate Level}

We have seen in Chapter 3 that the typical simulation run in this model exhibits three distinct phases (Fig. 5): the random phase, the growth phase, and the saturation phase. Over long time scales, these patterns tend to repeat themselves, as fully developed networks (that is, graph-spanning ACSs) undergo core-shifts and other transformations [10] that destroy the network supporting structure. This means that, despite these characterizations, the long term behavior of a single run is inherently random: the next big crash remains impossible to predict. We will refer to these individual runs as "microscopic" realizations. Opposed to that, in the following we will look at this system from a "macroscopic" point of view. This means that we will be interested in the aggregate behavior of a system characterized by its parameter $m$ when the simulation is repeated multiple times. Single runs ranging from $8 \cdot 10^{3}$ to $10^{6}$ time steps have been created, and ensembles of 100 runs analyzed. Each run starts from different initial conditions and random numbers. Thus, the computational resources used in these simulations were considerable, making simulations employing more than 500 nodes too time consuming to be realized. The data points for every instant in simulation time are averaged over the 100 microscopic runs. As we vary the system size $N$, the linking probability $p$ is rescaled inversely to $N$ so as to keep the average incoming connectivity $m=p \cdot(N-1)$ constant. Fig. 6 depicts a set of macroscopic runs for different $m$ values and varying system size.

To save computing time and resources, we adjusted the network evolution time $n$ to our needs in each case. Thus the $N=50$ system evolves over 8000 time steps, while the $N=500$ needs 40000 time steps to develop. To summarize our findings for the aggregate level, (i) we see that all of the curves are smooth and that none exhibit the kind of abrupt break-in that we observe in the microscopic runs. This represents the average evolutionary process of this system. That is, after the period of exponential growth of the average connectivity per node, the systems settle into a statistically stable condition of high average connectivity. These observations are valid for all of the studied system sizes and all values of $m$. We emphasize, (ii) that the transient is markedly different in the macroscopic run. For $m>0.15$ the initial random phase is almost in-existent in all systems. Instead, they enter the growth phase almost immediately, reaching saturation faster for larger $m$. The random phase is visible for smaller values of $m$ only. Eventually, we note (iii), that for large $m$ the saturation value of $\langle l\rangle$ is independent of system size $N$. That is, this system scales well with the number of nodes. In the limit

$$
\lim \left\{\begin{array}{l}
N \rightarrow \infty \\
p \rightarrow 0
\end{array}\right\} p \cdot(N-1)=\text { const }=m
$$



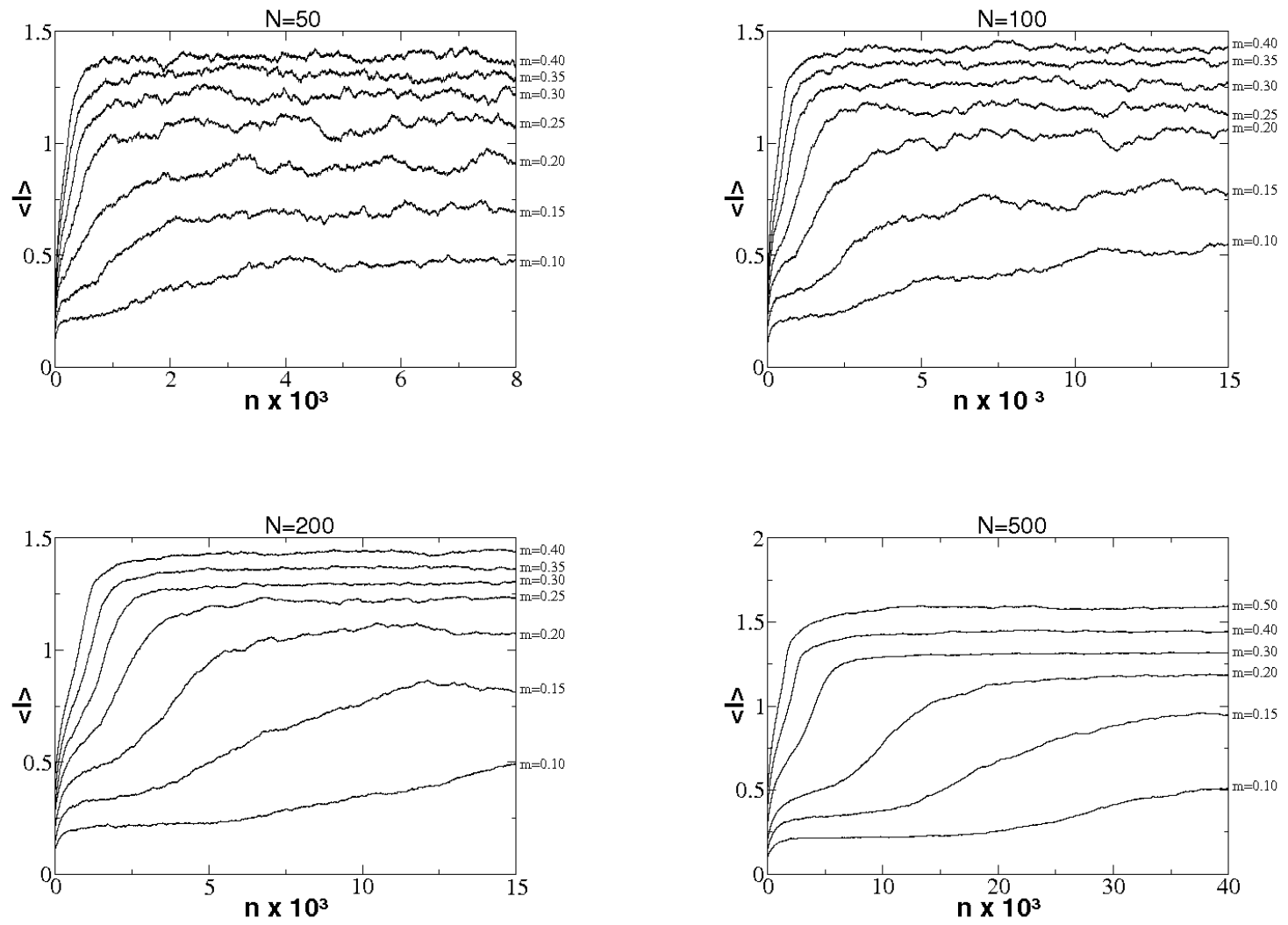

Figure 6: Average connectivity per node $\langle l\rangle$ after averaging over 100 runs. The different graphs represent different system sizes. top left: $N=50$. top right: $N=100$. bottom left: $N=200$. bottom right: $N=500$. Evolution time is chosen according to system size.

$\langle l\rangle$ is dependent only on $m$ as we will see below. In Fig. 7 we plotted the averaged values of $\langle l\rangle$ in the saturated regime as a function of $m$. We call this new variable $\langle l\rangle_{s}$. The values used here were computed by taking the average of $\langle l\rangle$ from every curve in Fig. (6) starting from a point at which saturation was deemed to have been reached. We then used a least squares method to fit a function to the data points that best approaches the qualitative shape of the data. As one can see from the plots, we find that $\langle l\rangle_{s}$ is a slow, monotonically increasing function of $m$, that can be well approximated by a function of the form

$$
\langle l\rangle_{s}(m)=a \cdot \ln (m)+b
$$

with the constants

$$
a=2.06 \pm 0.021 ; \quad b=0.66 \pm 0.042
$$


I.e., as a new finding observed on the aggregate level, we obtain a logarithmic scaling of the saturated average connectivity per node, $\langle l\rangle_{s}$ with $m$. Again, this scaling is independent of system size $N$ : both coefficients $a$ and $b$ vary only very slightly with $N$.

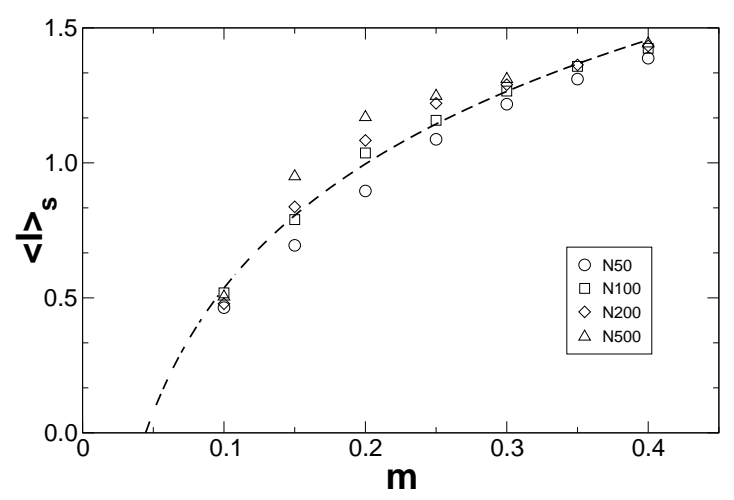

Figure 7: Saturated average connectivity per node $\langle l\rangle_{s}$ dependent on average incoming connectivity $m$. System sizes are the same as in Fig. 6. The plots are fitted by the logarithmic scaling of Eq. (10).

\section{Summary and Conclusion}

In this paper we have analyzed a model of network evolution that was recently introduced by Jain and Krishna [9] [10] as a combination of a hypercycle dynamics for the nodes and an external network dynamics for the links representing the catalytic interactions between the nodes. The basic concepts of the model are presented in Chapter 2. The population dynamics is described by Eq.(44), while the directed network of the species that catalyze each other are considered in the adjacency matrix $C$ in Eq. (6) . The evolution of the network is then governed by the natural selection of the weakest species and the introduction of novelty by new nodes. In such way a coupling of population dynamics with network dynamics is realized. In the first part of the paper, we have investigated the evolution of the network by means of single runs. The numerical implementation of the model is presented in some detail in Chapter 2.2. The crucial part of the Perron-Frobenius eigenvectors of the adjacency matrix $C$ in finding the attractor population for every network update step is emphasized. This option is used in the power method based on Eq. (8). In an iterative procedure the weakest species of the attractor is eliminated and replaced with a newly linked node. As a result of these computer simulations, Figs. 2 4 elucidate 
the structural changes of the network by means of different snapshots. They show typical cases of the phases of development of the network. Starting from an initial random phase the network processes to highly structured configurations. Complex structures evolve that can be characterized by Autocatalytic Sets (ACS). Our simulations validate the spontaneous occurrence and time evolution the ACSs described in [9]. Additionally we have computed the total number of links $l$ over network evolution time $n$ for different incoming connectivities $m$. In agreement with the findings in [9, 10] our simulations in Fig. [5 show an increase of $l$ for higher values of $m$ as well as crashes and recoveries of the evolving ACSs. The main new results are presented in Chapter 4, where the aggregate network dynamics is investigated. We have raised the question how the average connectivity per node $\langle l\rangle$, is influenced by varying values of the incoming connectivity $m$ and therefore analyzed multiple simulation runs. First we observed no abrupt break-ins in the average connectivity $\langle l\rangle$. This observance is opposite to the crashes we have seen for the supporting structures, the ACSs, during network evolution. After an exponential growth $\langle l\rangle$ saturates into a stable condition of high average connectivity. Second the saturation is reached for higher values of $m$. And third we have found a logarithmic scaling of $\langle l\rangle$ in Eq. (10) on $m$ for all system sizes $N$. Furthermore the saturation value of $\langle l\rangle$ is independent of $N$. There exist different ways to extend the basic model discussed in this paper. In [19] we have studied the impact of a selection mechanism on the performance of the system and its network structure by introducing a selection temperature and a performance threshold selection. We already found evidence for a critical value of this selection threshold for the global performance of the system. Moreover the threshold plays an important role in size and life span of the core of the ACS. Other future investigations of the model may involve the emergence of hierarchical organization resulting from the network evolution. Hierarchies are already discussed for different network topologies [25, 16, 23] and are also investigated in directed networks [24, 17]. The model discussed in this paper is seen as an agent-based model, where each agent represents a prebiotic chemical species. Agents are assumed as nodes of a network, where the links represent the catalytic interactions between the species. Because each node follows a deterministic eigendynamics, eq. (5), the model can be also regarded as a system of coupled differential equations. This however ignores the fact that the links (i.e. the couplings between the equations) change on a second time scale in a non-predictable way, because of the removal of the least fittest node and the random rewiring of a new node. Thus, from a methodological viewpoint, we rather address the model as an agent-based one. This perspective may hold regardless of the question whether the agents represent the population of a species, or individual entities, such as single companies in an economic setting. In fact, the catalytic network model discussed here, despite its simplicity, may serve as a good starting point for the study 
of the relationship between network structure and dynamics in several fields of research, in particular in the economic and social domain [22, 14, 8, 18]. For example, catalytic or hypercycle interaction can be used to model skills in economic organizations [15], or exchange of knowledge in innovation networks [12]. Using some more realistic rules for rewiring the network, one can then observe the emergence of a number of smaller ACS [12] instead of just one giant ACS in the current model. This affects the breakdown probabilities of the network consideraby. Cooperative networks related to the ones obtained from this model are also present in firm interactions. It was shown, for example, that financial systems form cooperative networks of ownership relationships with scale free properties [6].

\section{Acknowledgement}

The authors wish to thank Michael König (Zurich) for his help in redrawing Figs. 2,4, 6 and for comments on Chapters 1, 5.

\section{References}

[1] Bak, P.; Sneppen, K. (1993). Punctuated Equilibrium and Criticality in a Simple Model of Evolution. Physical Review Letters 71(24), 4083-4086.

[2] Berman, A.; Plemmons, R. J. (1994). Nonnegative Matrices in the Mathematical Sciences. Classics In Applied Mathematics, SIAM.

[3] Eigen, M. (1971). The selforganization of matter and the evolution of biological macromolecules. Naturwissenschaften 58, 465-523.

[4] Eigen, M.; Schuster, P. (1979). The hypercycle: A Principle of Self-organization. Berlin: Springer.

[5] Farmer, D. J.; Kauffman, S. A.; Packard, N. H. (1986). Autocatalytic Replication of Polymers. Physica D 22, 50-67.

[6] Garlaschelli, D.; Battiston, S.; Castri, M.; Servedio, V.; Caldarelli, G. (2005). The scale free nature of market investment networks. Physica A 350(2-4), 0310503491-499.

[7] Hofbauer, J.; Sigmund, K. (1984). Evolutionstheorie und dynamische Systeme. Verlag Paul Parey. 
[8] Holme, P.; Newman, M. E. J. (2006). Nonequilibrium phase transition in the coevolution of networks and opinions. Physical Review E 74, 056108.

[9] Jain, S.; Krishna, S. (1998). Autocatalytic Sets and the Growth of Complexity in an Evolutionary model. Physical Review Letters 81(25), 5684-5687.

[10] Jain, S.; Krishna, S. (2002). Large Extinctions in an Evolutionary Model: The Role of Innovation and Keystone Species. PNAS 99(4), 2055-2060.

[11] Kaneko, K. (2005). On Recursive Production and Evolvabilty of Cells: Catalytic Reaction Network Approach. Advances in Chemical Physics 130, 543-598.

[12] König, M.; Battiston, S.; Schweitzer, F. (2007). Microeconomic agent model of innovation networks. (to be submitted).

[13] Lee, D. H.; Severin, K.; Ghadiri, M. R. (1997). Autocatalytic Networks: the Transition from Molecular Self-Replication to Ecosystems. Current Opinion in Chemical Biology 1(4), 491-496.

[14] Miskiewicz, J.; Ausloos, M. (2006). Influence of information flow in the formation of economic cycles. In: M. Ausloos; M. Dirickx (eds.), The Logistic Map and the Route to Chaos, Berlin: Springer. pp. 223-238.

[15] Padgett, J. F. (1997). The Emergence of Simple Ecologies of Skill: A Hypercycle Approach to Economic Organization. In: W. B. Arthur; S. Durlauf; D. Lane (eds.), The Economy as an Evolving Complex System II, Addison-Wesley, vol. XXVII of Santa Fe Institute Studies in the Sciences of Complexity. pp. 199-222.

[16] Ravasz, E.; Barabasi, A.-L. (2003). Hierarchical Organization in Complex Networks. Physical Review E 67(2), 026112-1-7.

[17] Sánchez, A. D.; López, J. M.; Rodríguez, M. A. (2002). Nonequilibrium Phase Transitions in Directed Small-World Networks. Phys. Rev. Lett. 88(4), 048701.

[18] Schnegg, M. (2006). Reciprocity and the Emergence of Power Laws in Social Networks. International Journal of Modern Physics C 17(7), 1067-1076.

[19] Seufert, A.; Schweitzer, F. (2006). Threshold selection in an Evolutionary Network Model. (to be submitted).

[20] Stadler, P.; Fontana, W.; Miller, J. (1993). Random catalytic reaction networks. Physica D 63(3-4), 378-392. 
[21] Stadler, P. F. (1991). Dynamics of Small Autocatalytic Reaction Network IV: Inhomogeneous Replicator Equations. BioSystems 26, 1-19.

[22] Stauffer, D.; Hohnisch, M.; Pittnauer, S. (2006). The coevolution of individual economic characteristics and socioeconomic networks. Physica A 370(2), 734-740.

[23] Trusina, A.; Maslov, S.; Minnhagen, P.; Sneppen, K. (2004). Hierarchy Measures in Complex Networks. Physical Review Letters 92(17), 178702-1-4.

[24] Variano, E. A.; McCoy, Jonathan, H.; Lipson, H. (2004). Networks, Dynamics, and Modularity. Physical Review Letters 92(18), 188701-1-4.

[25] Vazquez, A. (2003). Growing network with local rules: Preferential attachment, clustering hierarchy, and degree correlations. Physical Review E 67(5), 056104-1-15.

[26] Wächtershäuser, G. (1990). Evolution of the First Metabolic Cycle. PNAS 87, 200-204. 